\title{
Compte-rendu de la table ronde « Justice spatiale dans les villes du Sud»
}

Colloque CNFG « La ville compétitive, à quel prix ? », 19 janvier 2012, Université de Paris-Ouest Nanterre

\section{Marianne Morange et Amandine Spire}

\section{(2) OpenEdition}

\section{Journals}

Édition électronique

URL : http://journals.openedition.org/cdg/1018

DOI : $10.4000 /$ cdg. 1018

ISSN : 2107-7266

Éditeur

UMR 245 - CESSMA

Référence électronique

Marianne Morange et Amandine Spire, « Compte-rendu de la table ronde « Justice spatiale dans les villes du Sud » », Carnets de géographes [En ligne], 4 | 2012, mis en ligne le 01 septembre 2012, consulté le 23 septembre 2020. URL : http://journals.openedition.org/cdg/1018 ; DOI : https://doi.org/ $10.4000 /$ cdg. 1018

\section{(c) (†) $\odot$}

La revue Carnets de géographes est mise à disposition selon les termes de la Licence Creative Commons Attribution - Pas d'Utilisation Commerciale - Pas de Modification 4.0 International. 


\title{
Compte-rendu de la table ronde « Justice spatiale dans les villes du Sud» Colloque CNFG «La ville compétitive, à quel prix ? », 19 janvier 2012, Université de Paris-Ouest Nanterre
}

MARIANNE MORANGE

Université Paris Diderot Géographe et urbaniste, SEDET, EA 4534 marianne.morange@,paris-diderot.fr

\author{
AMANDINE SPIRE \\ Université Paris Diderot \\ Géographe, SEDET, EA 4534 \\ amandine.spire@paris-diderot.fr
}

Ce carnet de lectures n'a pas pour point de départ la lecture d'un ouvrage mais un ensemble d'échanges ayant eu lieu lors d'une table ronde que nous avons organisée et animée en janvier 2012, à l'Université de Nanterre, à l'occasion du colloque international «La ville compétitive, à quel prix ? ( (organisé par le CNFG et le programme JUGURTA). Cette table ronde a réuni quatre membres du programme scientifique JUGURTA ${ }^{1}$ (justice, gouvernance urbaine et territorialisation dans les villes du Sud, financé par l'ANR «Suds » et dirigé par Philippe Gervais-Lambony) : Alain Dubresson (Professeur, Université Paris Ouest-Nanterre la Défense), Karine Ginisty (ATER à l'Université Paris-Est Marne-la-Vallée et doctorante à Paris Ouest-Nanterre La Défense), Susan Parnell (Professeur à l'Université du Cap, UCT) et Jean-Fabien Steck (Maître de conférences à l'Université Paris Ouest-Nanterre La Défense). Laurent Faret, Professeur à l'Université ParisDiderot et Directeur du SEDET, a joué le rôle de discutant externe au programme JUGURTA.

Pendant quatre ans (entre 2008 et 2012), le programme JUGURTA a donné la possibilité à une trentaine de chercheurs de travailler autour de questions de justice et d'injustice spatiale dans les villes du Sud. Cette table ronde a permis à ces géographes de confronter leurs démarches, leurs

\footnotetext{
${ }^{1}$ Nous signalons que nous avons également participé au programme JUGURTA.
} 
objets, leurs méthodes et leurs résultats et d'opérer un retour partiel sur leurs expériences individuelles de recherche et sur leur manière de mobiliser la notion de justice dans quatre villes africaines (Abidjan, Lomé, Le Cap, Maputo) affectées par des «problèmes de développement. Ils ont ainsi interrogé le rôle et le poids du Sud dans leurs manières d'aborder la notion de justice spatiale. L'objectif était d'interroger les conséquences et les attendus de leur participation à un programme sur la justice spatiale et les villes du Sud, en leur posant des questions communes : quand on traite de justice spatiale dans les villes du Sud, comment (et pourquoi) prendre en compte la spécificité de ces terrains? Qu'est-ce qu'une approche par le Sud peut apporter aux débats sur la justice spatiale? Quelles spécificités des enjeux de justice spatiale quand on parle du Sud?

S'il ne portait pas directement sur la compétitivité urbaine et l'entrepreneuralisme urbain, JUGURTA a néanmoins progressivement poussé les chercheurs impliqués dans ce programme à se positionner sur la question de la tension entre justice sociale et efficience économique, entre développement et redistribution. Cette question se trouve en effet au cœur des enjeux politiques dans bien des villes et elle prend un tour particulier dans les villes du Sud du fait des impératifs développementaux auxquelles ces dernières sont confrontées. Or la tension entre impératifs de justice et de compétitivité économiques offre de nombreuses possibilités d'élaboration d'un questionnement critique pour la géographie urbaine qui scrute les métropoles du Sud au prisme de la justice, notamment en ce qu'elle engage à reformuler des enjeux développementaux. C'est à ce titre que nous rendons compte des échanges de cette table ronde dans ce numéro.

La diversité des positionnements individuels a finalement fait émerger deux débats majeurs que nous espérons restituer au plus près de la parole des intervenants.

Justice spatiale et objets de recherches « développementaux », quelle relecture ?

Les chercheurs participant au programme JUGURTA ont considéré que la notion de justice spatiale appliquée aux espaces urbains appelait à réinterroger en priorité des formes d'injustice concrètes et matérielles somme toute assez classiques et bien connues dans les études portant sur le développement: les déséquilibres économiques et de services entre territoires ainsi que les inégalités sociales à différentes échelles. Les questions relatives au développement économique local, aux déguerpissements et d'accès au foncier, ou encore à la place du commerce de rue ont par exemple fédéré des discussions en partant de plusieurs cas d'études. Ces questions ont ici été réinterrogées en termes de justice. 
Travaillant sur la notion de métropolisation, A. Dubresson interroge les enjeux en terme de «bonne » gouvernance, d'efficacité gestionnaire ou de développement territorial compétitif. La construction de métropoles unifiées, au tournant des années 2000, dans le contexte postapartheid, visait avant tout à promouvoir un modèle de gouvernance apte à enclencher des redistributions fiscales et économiques entre territoires riches et townships ou camps de squatters délaissés sous l'apartheid, et à surmonter la fragmentation gestionnaire construite par l'apartheid. À travers la notion de justice spatiale, des perspectives comparatives Nord-Sud s'esquissent ici sur les liens entre stratégies de croissance territoriales métropolitaines et recherche de voies développementales locales.

À partir du cas de Maputo et dans le cadre d'une recherche doctorale, K. Ginisty questionne la notion de justice spatiale au prisme de l'étude des services urbains (un objet parmi d'autres possibilités selon elle). À travers la question de l'eau potable, elle s'intéresse surtout à la manière dont les citadins s'approprient, construisent et mobilisent les catégories du juste et de l'injuste dans le contexte post-socialiste du Mozambique. K. Ginisty a choisi de se démarquer des approches classiques sur l'eau potable au Sud, centrées sur l'accessibilité, la fragmentation des réseaux et la gouvernance.

Pour J-F. Steck, les notions de justice spatiale et de développement s'informent mutuellement. Le questionnement sur la justice spatiale, venu dans un second temps pour lui, après des travaux sur l'informalité à Lomé et Abidjan, ancrés dans le champ bien balisé des études développementales, l'a conduit à questionner la légitimité et la place du commerce de rue dans la ville africaine, et donc les relations entre informalité et espace public, en inscrivant ses travaux directement dans le champ des études urbaines. La justice spatiale permet là un glissement de point de vue intéressant.

Les questions identitaires et culturelles ont en revanche été relativement peu abordées au sein de JUGURTA dans la mesure où les chercheurs engagés dans ce programme n'étaient pas des spécialistes de ces questions, ni des questions de genre par exemple, mais des spécialistes des études urbaines qui privilégient une approche politique et sociale, souvent fondée sur une perspective matérialiste. La dimension « raciale» ou «minoritaire» de l'injustice en Afrique du Sud ou au Nicaragua par exemple, ainsi que les questions identitaires ont donc une place dans l'analyse de certains chercheurs, mais elles ne sont pas mobilisées comme des catégories d'analyse structurantes de la notion de justice spatiale par les chercheurs qui se sont exprimés dans cette table ronde. 
Ce constat ouvre un champ de recherche sur l'articulation entre ces approches théoriques et celles sur la subalternité ou le post-colonialisme, comme y invite le travail engagé collectivement sur les représentations de la justice, notamment dans l'ouvrage collectif issu de ce programme et actuellement en cours de publication ${ }^{2}$. A. Dubresson a ainsi signalé la piste de «l'atomisation des récits du juste et de l'injuste », liée aux micro-classifications raciales héritées en Afrique du Sud.

De ce point de vue, Karine Ginisty a rappelé le caractère très troublant de ses résultats sur la faible mobilisation des catégories de justice et d'injustice par les citadins de Maputo, qui renvoient à la nécessité d'une contextualisation locale et interrogent les capacités citadines de mobilisations politiques. Elle constate que les discours sur le juste et l'injuste sont confisqués par les acteurs publics, qui construisent des référents pour penser la justice et l'injustice alors même que les injustices vécues par les citadins au quotidien sont tus. La notion de justice spatiale lui a surtout servi de boîte à outils pour déchiffrer des dynamiques de pouvoirs en questionnant le sens des discours publics sur le développement et en interrogeant l'aspect normatif et axiologique de la notion de justice spatiale. La justice permet alors de repolitiser les approches du développement, marquées selon elle par une forte dérive techniciste. Il s'agit donc, à travers cette notion, de s'interroger non pas sur l'accessibilité matérielle à l'eau mais sur l'expérience de l'espace, de la ville, les pratiques sociales et le rapport politique à l'espace.

Les trois chercheurs, quel que soit leur rapport à la notion de développement, se rencontrent autour de la nécessité de situer les points de vue et de dépasser le caractère normatif de la notion de justice spatiale. Pour J-F. Steck, à Lomé, rechercher l'expression du juste et de l'injuste pose problème : il craint de projeter sur ce terrain ses propres représentations du juste et de l'injuste. Comme à Maputo, il n'existerait pas d'espaces d'expression politique propices à la contestation, surtout au niveau local. L. Faret rappelle d'ailleurs l'importance de l'historicisation des processus de construction des injustices et des rapports de domination, mais aussi des catégories du juste et de l'injuste, conformément à l'approche des savoirs situés. Egalement sensible à la spécificité des rapports de domination au Sud, il rejoint K. Ginisty pour mettre l'accent sur les vertus repolitisantes de la notion de justice spatiale qui permet selon lui de poser la question des dynamiques d'acteurs, des rapports de pouvoirs, notamment entre l'Etat (qui reste un acteur majeur au Sud malgré des capacités institutionnelles et financières souvent plus faibles qu'au Nord) et des acteurs transnationaux qui agissent à distance (notamment les investisseurs). Ces questions lui semblent centrales sur les terrains latino-américains dont il est spécialiste, où la question des

\footnotetext{
${ }^{2}$ GERVAIS-LAMBONY P., BÉNIT-GBAFFOU C., MUSSET A., PIERMAY J-L., PLANEL S., La Justice spatiale et la ville, regards du Sud, Paris, Belin.
} 
programmes d'ajustement structurel, du consensus de Washington, du mouvement justicialiste et de revendication de droits urbains sont au cœur des débats développementaux.

Une deuxième question a porté sur la spécificité des terrains nationaux pour penser la justice spatiale

Les intervenants ont en effet discuté de l'influence que certains Suds avaient pu avoir sur leur manière de penser la justice spatiale. Cette préoccupation était centrale pour la démarche comparative Sud-Sud qui fut celle du programme. Immédiatement, a surgi la question de la spécificité sud-africaine. Du fait des spécificités historiques, sociales et politiques de la trajectoire sud-africaine qu'a rappelées A. Dubresson (forte capacité de contrôle public, intensité du débat entre les gouvernements locaux et le monde académique sur l'espace, importance du thème de la réparation et de l'idéal de la transition démocratique ...), la question de la justice semble particulièrement légitime et pertinente dans ce contexte, à la fois du point de vue des pouvoirs publics qui s'en revendiquent et du point de vue des citadins qui réclament des formes plus nettes de redistribution et une transformation urbaine rapide et radicale. En outre, l'Afrique du Sud a joué le rôle de passeur d'une certaine bibliographie anglo-américaine et de thématiques moins abordées au Sud (la participation démocratique, la néolibéralisation, les échelles de gouvernance et de gouvernement...). A. Dubresson, qui a travaillé la question du développement local au Cap, souligne cependant la banalisation de ces enjeux et de cette trajectoire sud-africaine, et partant, sa capacité à dialoguer avec d'autres expériences de formulation de projets politiques « inclusifs ». Il retient l'Afrique du Sud comme un laboratoire pour analyser la circulation de modèles internationaux comme les City Improvement Districts ou les City Development Strategies.

À travers le débat sur le rôle de l'Afrique du Sud en tant que modèle régional ou que pays atypique, s'est engagée une discussion sur le sens de la justice spatiale au Sud. Le débat sur la spécificité sudafricaine ne s'est donc pas substitué à un débat général sur la justice dans le Sud global. Il l'a au contraire enclenché. Susan Parnell a ainsi rappelé comment pour elle, chercheure fortement engagée dans la réflexion politique et académique sur la refondation d'une ville post-apartheid juste au Cap, et dans la recherche d'une voie post-néolibérale, la justice spatiale est une notion pertinente pour penser des villes plus justes dans le Sud global, même si son contenu programmatique reste à définir. Elle souligne cependant que, quelles que soient les vertus et le caractère stimulant de cette notion, certaines spécificités des villes du Sud global rendent la définition d'un agenda unique (entre le Nord et le Sud) difficile : notamment la forte croissance démographique, la relative faiblesse des gouvernements locaux et des structures de gouvernance. La question de savoir si l'Afrique du Sud constitue un contrepoint et une exception, ou un modèle pour penser la justice spatiale sur le reste du continent (il est certain qu'elle affiche de telles 
ambitions politiques), voire au Sud, serait donc secondaire. Ce qui compterait avant tout serait l'ouverture d'un champ de réflexion propre à la recherche appliquée qui permettrait de dépasser le comparatisme interétatique (l'Afrique du Sud versus les autres pays) pour monter en généralité et penser la justice spatiale dans le Sud global.

Enfin, incidemment, S. Parnell s'est demandé pourquoi les villes occupent une place centrale dans les débats sur la justice spatiale, et s'il ne serait pas pertinent de réfléchir aux présupposés de cette orientation urbaine des agendas politiques, notamment au Sud. Rejoignant en partie ces préoccupations qui nous ramènent à la dimension développementale de la notion de justice, L. Faret souligne que, pour lui, le renouveau de la pensée sur ce qu'il préfère appeler les « inégalités territoriales », réside surtout dans un enrichissement des études développementales par l'intégration des apports des études urbaines. Si la rapidité des transformations des territoires et des espaces au Sud lui semble un phénomène ancien et bien connu, travailler la notion de justice spatiale permettrait surtout, selon lui, de mettre l'accent sur les dimensions intra-urbaines de ces mécanismes et du développement en général.

M. Morange \& A. Spire, 4 septembre 2012 